\title{
Alpha-heavy chain disease
}

INSERM

\section{Source}

INSERM. (1999). Orphanet: an online rare disease and orphan drug data base. Alphaheavy chain disease. ORPHA:100025

Alpha-heavy chain disease (alpha-HCD) is a type of HCD (see this term) characterized by the production of incomplete monoclonal alpha-heavy chains without associated light chains. Alpha-HCD is considered to be a subtype of immunoproliferative small intestinal disease (IPSID; see this term). The clinical presentation includes chronic diarrhea with evidence of malabsorption. 